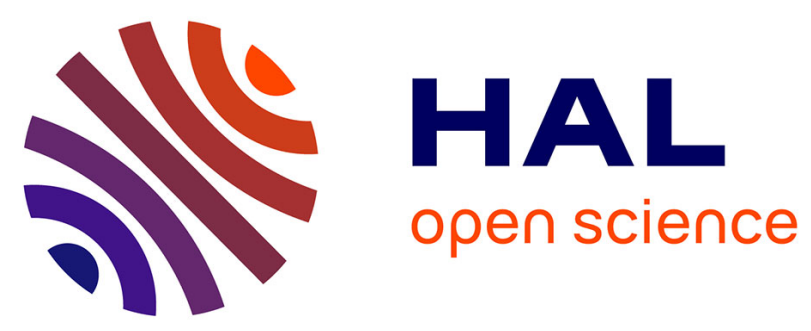

\title{
Comment articuler projets individuel, collectif et de territoire ? Le cas d'un collectif de transformation et commercialisation en circuits courts
} Émilie Lanciano, Marie Poisson, Séverine Saleilles

\section{- To cite this version:}

Émilie Lanciano, Marie Poisson, Séverine Saleilles. Comment articuler projets individuel, collectif et de territoire ? Le cas d'un collectif de transformation et commercialisation en circuits courts. Gestion 2000, 2016, 33 (2), pp.75-93. 10.3917/g2000.332.0075 . hal-01443955

\author{
HAL Id: hal-01443955 \\ https://hal.science/hal-01443955
}

Submitted on 23 Jan 2017

HAL is a multi-disciplinary open access archive for the deposit and dissemination of scientific research documents, whether they are published or not. The documents may come from teaching and research institutions in France or abroad, or from public or private research centers.
L'archive ouverte pluridisciplinaire HAL, est destinée au dépôt et à la diffusion de documents scientifiques de niveau recherche, publiés ou non, émanant des établissements d'enseignement et de recherche français ou étrangers, des laboratoires publics ou privés. 


\section{Comment articuler projets individuel, collectif et de territoire ? - le}

\section{cas d'un collectif de transformation et commercialisation en circuits}

courts $^{1}$

Article publié dans Gestion 2000, vol. 33 nº 2-3, p. 75-93, déc. 2016

LANCIANO Emilie, maître de conférences, Univ Lyon, UJM-Saint-Etienne, COACTIS, EA 4161, F-42023, SAINT ETIENNE, France 61)

POISSON Marie, Animatrice-coordinatrice FR Civam Bretagne

SALEILLES Séverine, maître de conférences, Univ Lyon, UCBL, LSAF, EA 2429, F-69007, LYON, France

\section{Résumé}

De nombreuses initiatives collectives impliquant des producteurs en circuits courts se développent : magasins et marchés de producteurs, plateformes d'approvisionnement de la restauration collective, ateliers collectifs de transformation, etc. Cependant, force est de constater que beaucoup de projets de ce type ne parviennent pas à émerger ou à se pérenniser. Mieux comprendre comment ces collectifs résolvent les différents paradoxes et difficultés auxquels ils sont confrontés constitue donc une attente forte.

Nous proposons une analyse de la trajectoire d'un collectif de transformation et de commercialisation en circuits courts, en montrant que la pérennité du système repose sur une articulation singulière et dynamique entre trois dimensions : le projet individuel, collectif et celui du territoire. A chacune des étapes de la vie du collectif, les acteurs mobilisent ces différents niveaux d'action afin de construire ou rétablir une cohérence et une dynamique.

\footnotetext{
${ }^{1}$ Ce travail a reçu le soutien du programme PSDR «Pour et Sur le Développement Régional» dans le cadre du projet LIPROCO. Nous remercions Fantine Olivier et Bernard Pecqueur pour l'encadrement du travail de collecte des données.
} 


\begin{abstract}
Many collective initiatives involving short supply chain's producers are growing up: shops and farmers' markets, collective outlets, logistic platforms, etc. However, many of such projects either fail to emerge, or to perpetuate themselves. Better understand how these collective strategies manage to solve the various paradoxes and difficulties they face, is therefore a strong expectation.

We analyze a collective strategy of farmers dedicated to the transformation of farmer's products and their commercialization. We show how the sustainability of its system is based on a dynamic relationship between three dimensions: the individual project, the collective project and the territorial project. At different stages of the collective project, the stakeholders mobilize these different levels of action in order to build or restore coherence and dynamics.
\end{abstract}

Mots-clés (5 max.) : circuits courts, collectifs, développement local, producteurs, stratégie 


\section{Introduction}

De nombreuses initiatives collectives impliquant des producteurs en circuits courts se développent : magasins et marchés de producteurs, plateformes d'approvisionnement de la restauration collective, ateliers collectifs de transformation, etc. Entre 2005 et 2013, les initiatives collectives en circuits courts se sont développées en Bretagne à un rythme de $+873 \%{ }^{2}$. Les atouts de l'action collective pour les producteurs, les filières et les territoires sont en effet nombreux (Mondy et Terrieux, 2011, Poisson et Saleilles, 2012, Mundler et Valorge, 2015) : maintien, développement et installation d'exploitations sur le territoire; diminution des coûts de production, développement de nouveaux débouchés ou de nouveaux potentiels de transformation; allègement des charges de travail ; création d'emplois (qualiticiens, bouchers, etc.); etc.

Cependant, force est de constater que beaucoup de projets de ce type ne parviennent pas soit à émerger, soit à se pérenniser. Dans une certaine mesure, les circuits courts sont une alternative au métier de producteur en coopérative agricole classique : ils sont un moyen pour insuffler un nouveau souffle entrepreneurial dans l'agriculture (Lanciano et Saleilles, 2010). Il s'agit, ainsi, de ne plus être assujetti à des donneurs d'ordres et de reprendre en main la commercialisation et la valorisation de la production et donc finalement la gestion de l'exploitation (Le Caro et Daniel, 2007). Pour autant, les producteurs en circuits courts ne sont pas isolés ; la valorisation en circuits courts implique la mobilisation et la formation de réseaux sociaux diversifiés : clients, groupes professionnels locaux, communauté locale, etc. (Dufour et Lanciano, 2012), qui sortent du champ classique de l'activité agricole. Par exemple, sur le cas des SCIC de filières courtes, Thomas (2009) montre qu'il faut dépasser le cloisonnement du monde agricole pour développer «une démarche coopérative où les agriculteurs et leurs organisations partageront des projets d'entreprise, non plus seulement entre agriculteurs, mais avec des salariés, des élus locaux ou associatifs, des entrepreneurs privés, etc.»(p.28). Mieux comprendre comment ces collectifs parviennent à

\footnotetext{
${ }^{2}$ Source : FRCIVAM Bretagne, Les circuits courts alimentaires en Bretagne : un état des lieux régional d'initiatives collectives pérennes, 2013, http://www.civambretagne.org/files/fil_bd/CC/Etatdeslieux_Circuits_courts_web.pdf
} 
résoudre les différents paradoxes et difficultés auxquels ils sont confrontés constitue donc une attente forte. Sur quelles bases se fondent ces nouvelles formes de coopération en circuits courts dans le monde agricole? Comment préserver la liberté d'action (notamment stratégie de commercialisation) de chaque exploitation en circuits courts, tout en développant un projet collectif autour d'un outil commun? Et comment maintenir cet équilibre projet du collectif / stratégie individuelle de l'exploitation tout au long de la vie du collectif?

Les approches en termes de stratégies collectives, particulièrement dans le cas des TPE (Très Petites Entreprises) montrent que l'articulation entre les stratégies individuelles des TPE et la stratégie collective constitue la principale difficulté (Bresser, 1988, Gundolf et al., 2006, Loup, 2007, Leyronas et Loup, 2008, Granata et Le Roy, 2014). En effet, à mesure que le projet se développe, des contradictions ou des ambiguïtés entre ces deux niveaux d'action mettent en péril l'engagement des acteurs et la dynamique collective. Nous proposons d'élargir ce système d'analyse en intégrant le territoire, dans ses différentes actions, à la fois comme un acteur de la dynamique collective (Sanz Canada et Muchnik, 2011, Fourcade, 2006, 2008) mais également comme une ressource (Gumuchian et Pecqueur, 2007) pour résoudre les tensions entre l'individuel et le collectif (Asselineau et Cromarias (2010, 2011). Ainsi, nous proposons que les projets collectifs en circuits courts reposent sur une articulation singulière et dynamique entre trois projets : le projet autour de l'outil, le projet des exploitations et le projet de territoire. Nous adoptons ici une vision dynamique de ce triptyque au travers de l'analyse fine d'un cas. Le Pré au Plat est une organisation collective de producteurs autour d'un outil de transformation et d'une commercialisation (individuelle et collective) en circuits courts qui fonctionne depuis près de dix ans. A travers cette analyse, nous souhaitons repérer comment le triptyque évolue au cours de la trajectoire du projet et quels sont les facteurs de stabilisation et de déstabilisant de l'équilibre entre les trois projets. 


\section{Collectifs de producteurs en circuits courts ou l'art d'articuler projets individuel, collectif et de territoire}

Afin d'analyser les collectifs de producteurs en circuits courts, nous mobilisons l'approche par les stratégies collectives de TPE qui nous permet de mieux identifier les mécanismes qui permettent d'articuler projets individuel et collectif (1.1.). Nous identifions le rôle du territoire comme ressource pour résoudre cette tension entre individuel et collectif (1.2.). Nous débouchons alors sur un triptyque de trois projets (individuel, collectif et de territoire) qu'il semble nécessaire d'articuler pour pérenniser les collectifs de producteurs en circuits courts.

\subsection{L'articulation entre projets individuel et collectif au cœur des stratégies collectives de TPE}

Les collectifs de producteurs en circuits courts se distinguent de la coopération agricole classique. Il ne s'agit pas de mettre en œuvre une stratégie de production, transformation et commercialisation commune à tous les producteurs membres, mais plutôt de faciliter l'accès des producteurs à des opportunités dont ils n'auraient pas pu bénéficier à titre individuel tout en leur permettant de garder la maîtrise de leur produit et de réaliser leur propre projet d'exploitation. En ce sens, ce sont des stratégies collectives entrepreneuriales (Leyronas et Loup, 2008) comparables à celles qui ont été étudiées dans le secteur du tourisme (Gundolf et al., 2006), de l'artisanat d'art (Loup, 2007) ou de la viticulture (Granata et Le Roy, 2014).

Les stratégies collectives constituent pour les Très Petites Entreprises (TPE), des voies pour pallier un certain nombre de difficultés qu'elles rencontrent (manque de ressources, par exemple). Elles permettent aussi de développer des actions en commun pour élargir leur champ des possibles en termes de marché, de client ou de produit (légitimer l'appartenance à un secteur, libérer une capacité d'entreprendre sur de nouveaux projets, etc.), voire pour construire, développer et protéger 
une identité commune (Loup, 2007). Ces stratégies sont fondées sur la mutualisation des ressources et le développement d'actions collectives (outil commun, actions de commercialisation, labels, etc.). Pour autant, les stratégies collectives de TPE ne sont pas dénuées de risques. Plusieurs facteurs peuvent venir perturber l'équilibre entre stratégie individuelle et stratégie collective (Leyronas et Loup, 2008) : des facteurs externes, des facteurs liés à la TPE (différences dans la place de la stratégie collective pour les entrepreneurs, augmentation des coûts de sortie) et des facteurs liés à la stratégie collective (différences de performances entre les entreprises, entrée/sortie de nouveaux, évolution du contenu de la stratégie collective). Leyronas et Loup (2008) considèrent que ce déséquilibre permanent est lié au fait que les stratégies collectives de TPE relèvent d'une quadruple ambiguïté : une ambiguïté d'organisation car il s'agit de systèmes organisant incomplets, sans hiérarchie, ni contrôle afin notamment de permettre aux partenaires d'avoir des attentes qui ne sont pas nécessairement identiques ; une ambiguïté d'intention dans la mesure où les objectifs à court et moyen terme poursuivis ne sont pas les mêmes selon les entrepreneurs; une ambiguïté de compréhension car les entrepreneurs n’ont pas nécessairement perçu de prime abord les changements auxquels la participation à une stratégie collective allait les conduire y compris dans le fonctionnement de leur propre entreprise ou dans la manière dont les décisions se prennent; une ambiguïté d'histoire liée au fait que ces stratégies se développent le plus souvent en TPE entre des entreprises appartenant à un territoire, entre des entrepreneurs qui se connaissent souvent depuis longtemps.

Le management de stratégies collectives de TPE est donc délicat (Granata et Le Roy, 2014). Il s'agit en effet de concevoir un système de coordination qui permette la conciliation des stratégies individuelles et collectives (Bresser, 1988) et, à terme, l'intégration des objectifs collectifs aux objectifs individuels des TPE (Gundolf et al., 2006). Dans le cas des stratégies coopétitives (entre concurrents), Granata et Le Roy (2014) montrent que ce management repose sur un double principe : séparation organisationnelle (via la création d'une structure dédiée) entre la compétition et la coopération et intégration du paradoxe coopétitif par le dirigeant de la TPE. La pérennité d'un 
tel système de coordination nécessite aussi la création d'une communauté socio-économique entre les dirigeants ; le collectif ne peut donc être exclusivement à visée sociale ou à visée économique (Gundolf et Jaouen, 2010). En effet, les relations amicales facilitant la naissance de relations d'affaires, relations qui ne peuvent perdurer sans mettre en place des relations personnelles de confiance, les relations interpersonnelles sont particulièrement importantes à la réussite de telles stratégies interorganisationnelles (Gundolf et Jaouen, 2008). Par ailleurs, l'intervention d'un acteurtiers peut faciliter l'équilibrage entre stratégies individuelle et collective (Loubaresse et Pestre, 2012). Ainsi, l'intervention d'une institution, en tant qu'acteur extérieur au groupe, évite parfois les conflits d'intérêts entre les entreprises partenaires (Gundolf et Jaouen, 2009). Effectivement, dans les collectifs de producteurs en circuits courts, interviennent différents acteurs-tiers, comme les institutions du territoire, des acteurs de l'accompagnement, etc. C'est pourquoi, nous proposons de considérer le territoire comme une ressource pour résoudre la tension entre individuel et collectif.

\subsection{Le territoire comme ressource pour résoudre la tension entre projets individuel et collectif}

Les stratégies collectives de TPE sont souvent construites sur une base territoriale. Le territoire y est pensé comme un espace d'action et de coordination (cf. par exemple, l'ambiguïté d'histoire évoquée par Leyronas et Loup, 2008), la proximité géographique favorisant d'autres formes de proximités entre acteurs. Il peut aussi être vu comme une ressource de valorisation, via la mobilisation de ressources territoriales, c'est à dire de ressources pour lesquelles le contexte sociospatial contribue directement à la valeur de ce qui est produit (Gumuchian et Pecqueur, 2007). Par exemple, les Systèmes Alimentaires Localisés (SYAL) permettent la mise en valeur (souvent via des certifications de qualité territoriale) de produits alimentaires typiques, de terroir, identitaires, etc. (Sanz Canada et Muchnik, 2011) ou choisissent de s'appuyer sur un espace naturel géographique et/ou économique, pour en faire une image valorisante de leur activité (Fourcade, 2008). 
Contrairement aux SYAL, les collectifs de producteurs en circuits courts ne visent pas forcément à valoriser des productions spécifiques ou à mettre en valeur un patrimoine culinaire, alimentaire et/ou agricole particulier. Plus qu'un espace de coordination ou qu'une ressource de valorisation, il est en effet possible de considérer le territoire comme un acteur à part entière de ces stratégies collectives de TPE. Asselineau et Cromarias (2010) proposent le concept de « stratégie collective de territoire » pour qualifier des stratégies collectives où, au terme d'un processus progressif d'institutionnalisation et de formalisation d'une stratégie (au départ purement entrepreneuriale) par le biais de l'implication des acteurs publics, les visions des dirigeants d'entreprise et des élus locaux convergent. Ce type de stratégie ne peut fonctionner qu'à la double condition que 1) les entrepreneurs, au-delà de la création de richesses, aient l'ambition de participer au développement de leur territoire et que 2) les acteurs publics voient dans le développement des entreprises l'intérêt de la collectivité. Si l'alternance de processus entrepreneuriaux et institutionnels est identifiée comme un élément clef de la pérennisation des stratégies collectives locales, l'implication éventuelle d'une institution ne doit pas être trop forte afin d'éviter un boycott entrepreneurial (Gundolf et al. 2006). Ainsi, Fourcade (2006) montre que la variable territoriale, incarnée par ses institutions, s'intègre différemment selon les stratégies collectives : les collectivités territoriales peuvent être de véritables mentors de la démarche, ou être de simples interfaces dans la gouvernance (avec un degré d'influence sur les entreprises plus ou moins fort). Asselineau et Cromarias (2011), eux-mêmes, constatent que l'émergence d'une «stratégie collective de territoire » ne va pas de soi. Ainsi, même si les entreprises ont économiquement intérêts à coopérer et si une telle coopération fait l'objet de politiques territoriales actives, elle peut se heurter à l’hostilité des entreprises concernées.

Pour éviter un tel « boycott entrepreneurial» (Gundolf et al., 2006), il apparaît indispensable que le projet de territoire soit articulé à la fois avec le projet collectif mais également avec les projets individuels de chaque entreprise. Par exemple, dans le cas des SYAL, Fourcade (2008), les 
entreprises ancrées dans leur territoire/terroir l'utilisent comme image valorisante et visualisable du SYAL, notamment pour exporter leurs produits.

C'est pourquoi, nous proposons de considérer les collectifs de producteurs en circuits courts comme étant au centre d'un triptyque de projets : individuel, collectif et de territoire.

\section{Figure 1 - Le triptyque des projets des collectifs en circuits courts}

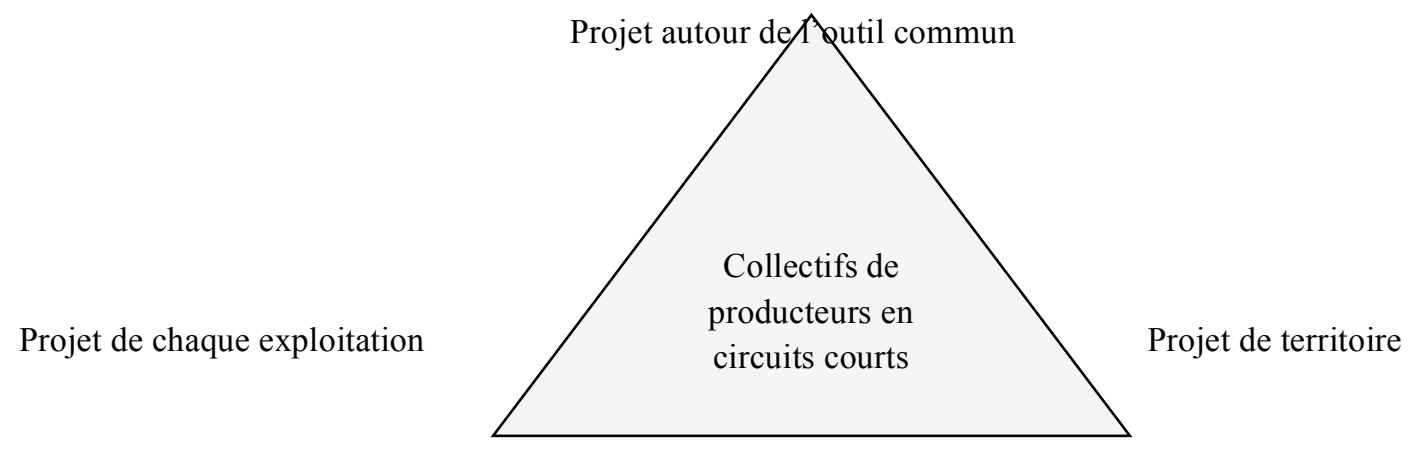

Une fois l'existence de ce triptyque constaté, reste à définir la façon dont ces trois projets s'articulent et ce, sans rester dans une approche statique de repérage des facteurs d'équilibre et de déséquilibre, mais en considérant que le mince équilibre entre ces trois projets évolue tout au long de la vie du collectif. Nous proposons de réaliser l'étude d'un cas de collectif de producteur en circuits courts en voie de pérennisation afin d'analyser l'évolution et l'articulation du triptyque tout au long de la vie de ce collectif. 


\section{2. «Du Pré au plat », un collectif de producteurs en circuits courts en voie de pérennisation}

Après avoir justifié le recours à une étude de cas unique (2.1), nous présentons en quoi celle que nous avons choisie, «Du pré au plat» est exemplaire pour questionner l'articulation des projets individuels, collectif et de territoire (2.2).

\subsection{Le choix d'une étude de cas unique}

La méthodologie retenue est l'étude de cas unique (Yin, 1994), l'objectif étant d'explorer des pratiques ou des phénomènes nouveaux (Wacheux, 1996). Nous avons effectué en 2010, 11 entretiens semi-directifs, dont 9 auprès de producteurs (cf. Tableau 1 qui présente la variété des enquêtés). Les enquêtés étaient invités à apporter un regard réflexif sur leur implication dans le projet collectif à différentes étapes. Chaque entretien d'une durée de 1 h30 à $3 \mathrm{~h}$ a été intégralement retranscrit. L'implication familiale de l'enquêtrice a facilité la prise en main du contexte et la collecte des données. Elle implique également une certaine part de subjectivité pour l'analyse. Une triangulation de ces données est assurée par l'étude de documents secondaires : compte-rendu de réunions et de voyages d'études, règlement intérieur de l'atelier, dossiers de demandes de subventions.

\section{Tableau 1. Caractéristiques des enquêtés}

\begin{tabular}{|c|c|c|c|}
\hline Statut des enquêtés & Date d'entrée dans le projet & Responsabilités dans le projet & Caractéristiques des exploitations \\
\hline 9 producteurs & $\begin{array}{l}2000 \text { 's : } 4 \text { producteurs } \\
2002: 3 \text { producteurs (sortie } \\
\text { envisagée pour l'un en } 2010 \\
\text { puis abandonnée) } \\
2005: 1 \text { producteur } \\
2009: 1 \text { producteur }\end{array}$ & $\begin{array}{l}8 \text { fondateurs de la SARL et de la CUMA: } \\
\S \text { dont } 4 \text { membres du comité de gestion } \\
\text { de la SARL } \\
\S \text { dont le Président de la CUMA, le } \\
\text { Président de l'Association des Eleveurs, le } \\
\text { Président de l'Association pour le projet } \\
\text { d'abattoir } \\
1 \text { adhérent uniquement à la CUMA }\end{array}$ & $\begin{array}{l}\text { Statut des exploitations: } \\
3 \text { GAEC, } 3 \text { EARL, } 2 \text { exploitations individuelles } \\
\text { Nature des productions : } 2 \text { viande bovine, } 3 \text { laitières, } \\
2 \text { viande ovine, } 1 \text { porcs, } 1 \text { volailles }\end{array}$ \\
\hline 1 accompagnateur & \multicolumn{3}{|c|}{ Depuis 2000's pour suivre le projet et depuis 2003-2004 comme accompagnateur } \\
\hline 1 salariée & \multicolumn{3}{|c|}{ Suit le projet depuis 2000's, embauchée comme salariée-gérante (2006-2014) } \\
\hline
\end{tabular}


Les éléments collectés ont donné lieu à une analyse intra-cas qui présente l'outil collectif (ses spécificités, son fonctionnement, ses membres), sa trajectoire (motivations individuelles et objectifs communs lors du projet initial, montage du projet, historique vécu, perspectives), les difficultés, la répartition des rôles et management (charge de travail, délégation, engagement), la gouvernance (implication, prise de décision, rapport à l'innovation, au projet collectif) et les attentes sur l'accompagnement. Elle tient compte du biais des récits rétrospectifs (freins psychologiques à se remémorer des faits passés notamment) via la recomposition des évènements et le croisement des discours. Cette étude vise à comparer les récits des différents producteurs partie-prenante du projet pour identifier les évènements qui ponctuent la trajectoire du projet et analyser les récurrences et les points de tensions à chaque étape.

Dans un souci de validation, nous avons soumis à relecture la synthèse finale, retraçant la trajectoire du projet, à l'accompagnateur et à un des producteurs enquêtés. Par ailleurs, les échanges téléphoniques et le recueil de données informelles avec ces deux enquêtés se sont poursuivis jusqu'à aujourd'hui.

\section{2.« Du pré au plat », un cas exemplaire d'articulation des projets individuels, collectif et de territoire}

« Du pré au plat » (nom fictif) est une initiative lancée à proximité d'une grande agglomération de l'ouest de la France par 10 producteurs en circuits courts. La démarche a été initiée, il y a presque vingt ans, et a débouché à ce jour sur la création de deux structures collectives: une CUMA (en 2004) qui propose des prestations de découpe et transformation dans un atelier multi-espèce (bœuf, porc, veau, agneau, volaille) à une centaine d'exploitations de la région et une SARL (en 2006) qui s'occupe de la commercialisation des produits des producteurs fondateurs via un magasin et de la vente aux professionnels (traiteurs, restaurateurs, cantines scolaires, supérettes, etc.). Le service global de valorisation des produits alimentaires locaux (comprenant la commercialisation) n'est 
donc pas destiné à tous les producteurs utilisateurs de la CUMA mais implique un engagement majeur de la part des 10 associés de la SARL. Le chiffre d'affaires cumulé de la SARL et de la CUMA est d'un million d'euros en 2013.

La CUMA propose aux éleveurs d'organiser le transport de leurs animaux vivants au départ de l'exploitation, puis en carcasse entre l'abattoir et l'atelier de découpe, jusqu'au retour des caissettes à la ferme. A chaque étape, le produit appartient à l'éleveur. Il peut d'ailleurs intervenir pour découper ou transformer sa viande (mise à disposition du matériel et des salles) ou bien, bénéficier des savoir-faire des bouchers professionnels. L'objectif est de permettre aux éleveurs de maîtriser la valeur ajoutée de leurs produits: "l'outil doit répondre à un besoin sur l'exploitation et le producteur est acteur de sa propre stratégie » (accompagnateur). Il s'agit donc de permettre la réalisation de la stratégie individuelle de chaque exploitation. Pour autant, dans un contexte de concurrence locale forte (grosses entreprises privées de découpe), il s'agit aussi de transformer les utilisateurs de la CUMA en véritables parties prenantes d'un projet collectif. En effet, si les exigences qualité et le portage du projet sont des critères de choix importants d'un prestataire, le prix reste décisif. L'absence de projet collectif autour de l'activité de transformation est donc perçue par les fondateurs comme un risque. Aussi, ils cherchent à mettre en place des formations sur la découpe proposées aux producteurs, à mobiliser les coopérateurs via des débats sur les recettes, à développer des «commissions matériels» (suivi, entretien, innovation technique). Toutefois, le projet collectif des 10 exploitations fondatrices s'incarne essentiellement au travers de la SARL. En effet, la question de la commercialisation pousse les producteurs, au-delà des débats techniques, à s'engager vers des débats stratégiques : circuits vers lesquels aller, délimitation de l'aire de chalandise, etc.

Aujourd'hui, «Du Pré au Plat» est le moteur d'une gouvernance alimentaire territoriale en construction. Fondé par 10 producteurs, l'outil offre des services à l'ensemble des producteurs du territoire. En 2014, les associés de la SARL et les adhérents de la CUMA emploient une dizaine de salariés (bouchers, opérateur d'emballage, agent d'entretien) dans un souci de dynamiser l'emploi 
local. « Du Pré au Plat » est mobilisé par les collectivités locales dans la promotion du territoire : attractivité économique pour des investisseurs, image d'une dynamique agricole durable et d'une alimentation de proximité, etc. « Du Pré au Plat» diversifie actuellement ses activités (logistique et projet d'abattoir) permettant à d'autres acteurs du système alimentaire local comme les artisansbouchers, jusqu'alors plutôt concurrents, d'intégrer le projet et une modification du statut juridique vers une SCIC (Société Coopératives d'Intérêt Collectif) est envisagée.

\section{L'articulation des projets de «Du pré au plat » dans la construction d'une stratégie collective de territoire}

Nous décrivons la trajectoire de développement de « Du pré au plat » en trois phases (cf. tableau 2). Si la phase d'émergence se focalise essentiellement sur l'articulation du projet collectif aux projets des exploitations (3.1.), elle débouche sur une phase de remise en cause où les déséquilibres entre projets individuels et collectif sont exacerbés (3.2.). Nous montrons alors comment l'articulation au projet de territoire a permis de résoudre ces tensions et d'opérer un glissement vers une stratégie collective de territoire (3.3.). 
Tableau 2. Evènements clés dans la trajectoire du projet du Pré au Plat

\begin{tabular}{|c|c|c|}
\hline & Événements du Pré au Plat & Événements contextuels et/ou locaux \\
\hline Emergence & 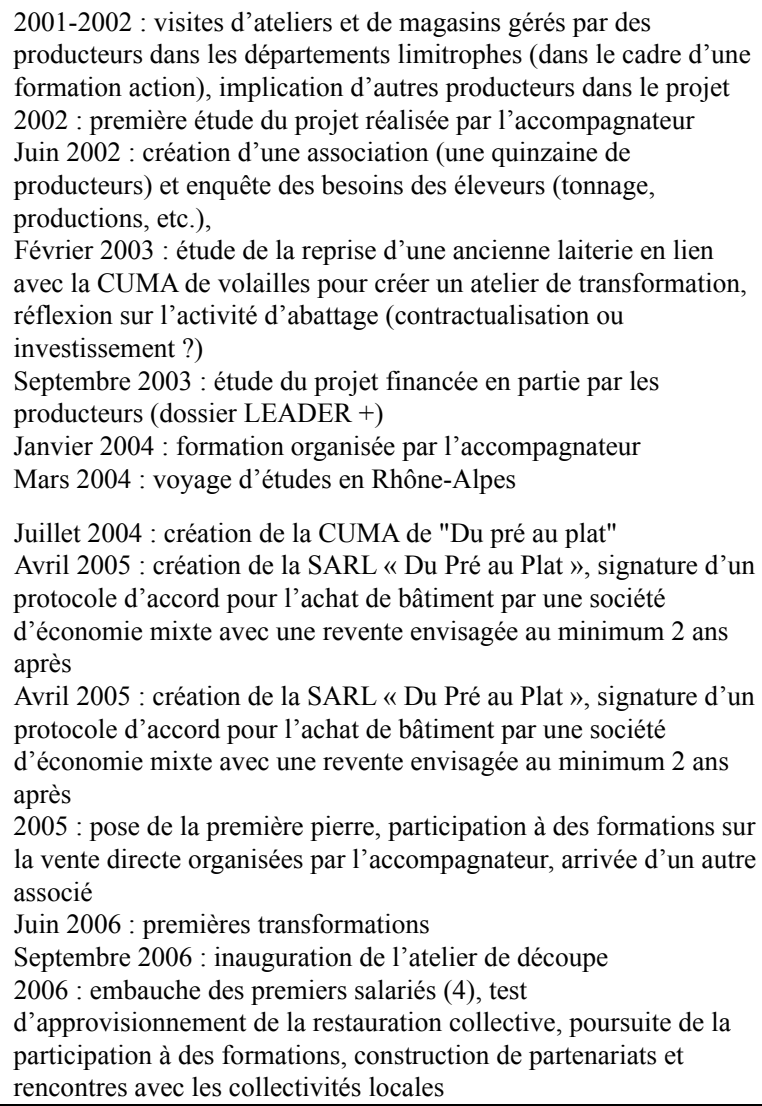 & $\begin{array}{l}2000 \text { : Crise de l'ESB pour la viande bovine, } \\
\text { accentuation des cirses sur le marché des productions } \\
\text { hors sols, modification des habitudes alimentaires, } \\
\text { dégradation des prix sur le marché } \\
2001 \text { : création d'une CUMA pour un projet d'abattage } \\
\text { de volailles (certaines exploitations sont également } \\
\text { engagées dans le projet du Pré au Plat) } \\
2002-2003 \text { : réflexion autour des CTE } \\
\text { Seconde crise de la viande bovine }\end{array}$ \\
\hline Remise en cause & $\begin{array}{l}\text { Octobre } 2007 \text { : intervention de la Chambre des Métiers pour } \\
\text { répondre aux problèmes de main d'œuvre (répartition des tâches, } \\
\text { organisation, charges ...) } \\
2008 \text { : réflexion sur les stratégies à adopter (salariales et } \\
\text { commerciales) ; apport d'un nouveau capital par les associés ; } \\
\text { embauche de nouveaux salariés ( } 3 \text { ) } \\
2009 \text { : des coopérateurs quittent le groupe (coût des prestations trop } \\
\text { élevé) } \\
2010 \text { : premier exercice positif (sur l'année 2009), un fondateur } \\
\text { envisage de quitter le groupe } \\
2011 \text { : exercice négatif (sur l'année 2010), test de transformation de } \\
\text { produits secs }\end{array}$ & $\begin{array}{l}2010 \text { : lancement d'une opération « Terroirs sur la } \\
\text { route » }\end{array}$ \\
\hline Renouvellement & $\begin{array}{l}2012 \text { : exercice positif (sur l'année 2011) } \\
\text { Janvier } 2012 \text { : création d'une association pour le projet d'abattoir } \\
2012 \text { : arrivée de nouveaux producteurs, perspectives de rachat des } \\
\text { bâtiments par la SARL, expérimentation de la mutualisation de } \\
\text { livraisons (projet intitulé Terroirs sur la route, porté par la CUMA) } \\
2013 \text { : développement de partenariats avec les bouchers-charcutiers } \\
2014 \text { : changement du mode de gestion (co-gestion assurée par } \\
\text { deux éleveurs associés, départ de la salariée gérante) }\end{array}$ & 2012 : cessation d'activité d'un autre atelier de découpe \\
\hline
\end{tabular}




\subsection{Phase d'émergence : l'articulation du projet collectif aux projets des exploitations, moteur de l'équilibre}

La réflexion autour d'un atelier de découpe date de la fin des années 90 et est portée par un groupe, issu d'une dynamique collective et syndicale forte, d'une dizaine de producteurs, "relations de voisinage, relations professionnelles, rencontres politico-syndicales », dont les motivations sont doubles : «chercher des plus-values» et «dynamiser le territoire»(Gilbert, éleveur de viande bovine et volailles). On retrouve donc dès le démarrage deux ingrédients favorables : des relations interpersonnelles fortes (Gundolf et Jaouen, 2008), amicales ou liés au partage de la même vision de l'agriculture et une volonté chez ces entrepreneurs, au-delà des gains économiques de participer, via leur projet commun, au développement du territoire (Asselineau et Cromarias, 2010). Cette réflexion trouve un cadre institutionnel ${ }^{3}$ qui permet alors de réunir des représentants professionnels et élus locaux mobilisés autour de la valorisation des productions locales et de bénéficier de fonds pour des études de préfiguration. Si le groupe "tourne rapidement en rond», c'est un voyage d'étude qui va permettre de forger le groupe et de prendre de véritables décisions. "ça a été un acte où d'ailleurs certains se sont retirés. Et c'est là que le groupe des 10 s'est créé » (accompagnateur). Bien que la création d'un abattoir ait été un temps envisagée, c'est une autre option qui a été retenue. La CUMA de transformation est créée en 2004. L'implication des collectivités territoriales (communauté de communes, conseil général) permet l'implantation du site dans une zone d'activité et la réalisation des investissements ${ }^{4}$ (subventions, garantie d'emprunt). «Sur des projets comme ça, on ne peut pas imaginer que les collectivités territoriales au moins en termes d'influence ne soient pas omniprésentes » (accompagnateur). L'implication du département est facilitée par un changement de majorité faisant du projet un véritable enjeu politique et de

\footnotetext{
${ }^{3}$ Le choix a été fait, sur le canton, de rentrer sur la dynamique de CTE (Contrat Territorial d'Exploitation entre l'Etat et l'exploitation) par l'entrée circuits courts

${ }^{4}$ De par cet appui des collectivités, les producteurs fondateurs n'ont apporté, au démarrage, qu'un capital modeste ( $1000 €$ chacun sur un investissement total de $800 \mathrm{~K} €$ ), ils ont en revanche dû réinvestir par la suite.
} 
développement local (projet de 10 producteurs pour 150, embauche de salariés, approvisionnement de cantines scolaires, etc.). Ayant fait «le choix d'avoir un outil de transformation de capacité technique et de production important», il s'agit de rentabiliser cet outil, de "développer une stratégie pour pouvoir rentabiliser l'emploi », et donc de l'ouvrir à un ensemble plus large de producteurs du territoire. «Ça n'a de sens que si demain on est 150 » (accompagnateur). La convergence des visions entre entrepreneurs et élus locaux (Asselineau et Cromarias, 2010) est donc forte dès le démarrage du projet.

En 2005-2006, les étapes marquantes se succèdent: pose de la première pierre, création de la SARL, accord avec une société d'économie mixte pour l'achat des bâtiments, inauguration en présence des élus, premières transformations de produits, etc. Dès 2006, des questions organisationnelles se posent: salariat, règlement intérieur, répartition des tâches, etc. Les producteurs embauchent plus de main d'œuvre que prévue initialement : 2 bouchers, une opératrice emballage, une gérante. Aussi, la phase de naissance sera courte car rapidement, le groupe moteur s'engage dans une dynamique tout azimut: innovation de produit, recherche de débouchés, multiplication des animations, absorption des volumes. «A cette période, ils ont foncé tête baissée » (accompagnateur). C'est aussi une période où la SARL établit ses premiers partenariats avec les collectivités. Le conseil général pousse le groupe à s'investir dans la restauration collective et notamment approvisionner les cantines scolaires de la seconde agglomération du département.

Dans cette phase d'émergence, l'équilibre s'appuie surtout sur les relations entre le projet collectif et celui des exploitations. Cet équilibre constitue d'ailleurs l'argument primordial pour faire adhérer un grand nombre de producteurs au projet et pour rallier aussi les politiques à la cause agricole locale. Le projet collectif a d'abord pour objectif de développer un outil technique commun qui devrait permettre aux agriculteurs de résoudre certains problèmes de coûts et leur donner l'accès à de nouveaux marchés. Si certains fondateurs souhaitent avoir un lien avec le développement territorial à long terme, le territoire n'intervient à ce stade qu'au travers de sa dimension 
institutionnelle et du soutien des collectivités territoriales. Ainsi, l'équilibre entre les trois projets peut être schématisé de la sorte.

\section{Figure 2 - L'articulation des projets durant la phase d'émergence}

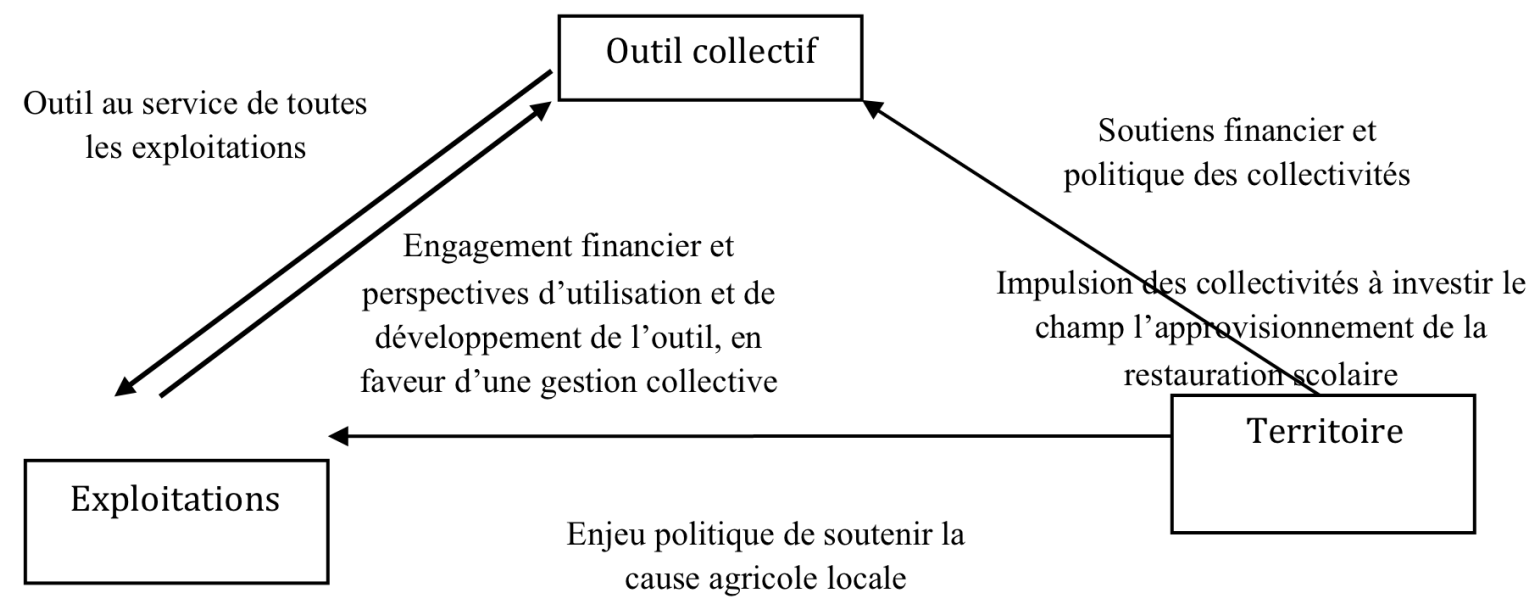

Comme le note Leyronas et Loup (2008), la cohérence entre le projet individuel et le projet collectif constitue une difficulté majeure dans la mise en œuvre d'une stratégie collective entrepreneuriale. Cette difficile articulation est de surcroit amenée à être reconsidérée, à mesure que l'activité collective se développe. C'est ce qui va rapidement se produire pour le cas de « Du pré au plat », les difficultés économiques et de coordination apparaissant.

\subsection{Phase de remise en cause : l'exacerbation des déséquilibres projets d'exploitation / projet collectif}

Certains producteurs sonnent l'alarme dès 2007-2008. De fait, des facteurs macroéconomiques comme la hausse du carburant mais également une difficulté à anticiper et planifier les coûts et les recettes du projet ont fortement affecté les prévisions de résultats et obligé à procéder à de nouveaux investissements de la part des producteurs. Certains producteurs commencent à exprimer une méfiance à l'égard du projet : un budget qui explose au regard du prévisionnel, une stratégie commerciale qui s'éloigne de leurs attentes, une implication hors de mesure, etc. Des tensions 
émergent entre la gérante et un associé, entre les associés et les salariés, etc. L'équilibre entre projet collectif et stratégie des exploitations est alors fragilisé par le départ de certains (qui n’y croient pas, qui n'en veulent pas). Beaucoup s'en détachent s'impliquant de moins en moins dans les tâches collectives alors que d'autres s'investissent à fond, quitte à délaisser leur propre activité sur l'exploitation.

L'affaiblissement de cette relation conduit à la remise en cause de l'existence de la CUMA et d'un projet collectif et humain autour de l'outil. Les producteurs-associés prennent note de ce déséquilibre et continuent de penser que cette interdépendance est indispensable mais peinent à trouver des solutions directes et immédiates dans cette relation. Pour augmenter les volumes, deux ou trois producteurs très engagés se lancent dans des opérations de démarchage des producteurs et de la restauration collective. Ces actions donneront lieu notamment à l'élaboration de prestations individualisées pour les producteurs : transports et/ou découpe et/ou transformation, participation du producteur à certaines activités pour réduire les coûts, etc. Mais les résultats sont maigres, le groupe tâtonne. Les producteurs passent plus de 3 ans à effectuer des aller-retours entre des expérimentations pour rechercher une certaine opérationnalité.

Figure 2 - L'articulation des projets durant la phase de remise en cause

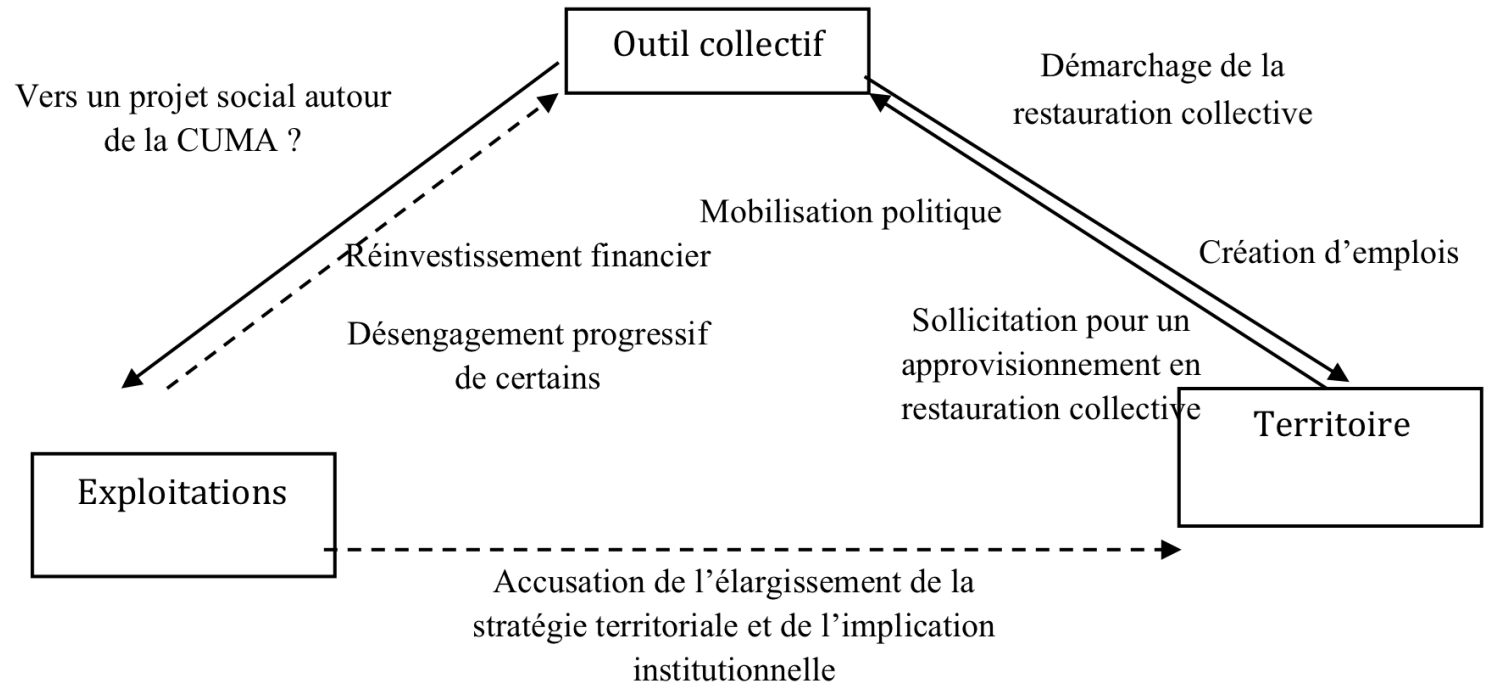


Ces difficultés d'articulation entre l'outil collectif et les exploitations sont en partie résolues en convainquant l'argument du territoire comme justification au projet collectif. Effectivement, le territoire est mobilisé, non plus seulement au travers de l'acteur institutionnel, mais également dans ses dimensions socio-économiques au travers des potentialités de développement de l'activité qu'il représente. La relation entre le projet collectif et le territoire s'enrichit et s'établit donc à double sens : il constitue une ressource pour les producteurs qui démarchent la restauration collective, mais également pour les politiques qui mettent en avant l'outil dans leur politique de développement économique local. L'ouverture au territoire constitue alors un outil de stabilisation pour un retour à l'équilibre entre exploitations et outil collectif.

\subsection{Phase de renouvellement, vers un équilibre du triptyque par un renforcement de la convergence outil collectif / territoire.}

Malgré ces difficultés, un nouvel élan s'observe en 2011-2012. «Du pré au plat » entre alors dans une phase de renouvellement, où on observe un rééquilibrage du triptyque par la recherche d'une convergence plus grande entre projet collectif et projet de territoire. En effet, les collectivités vont progressivement mobiliser l'outil dans la promotion de leur territoire : attractivité économique pour des investisseurs, image d'une dynamique agricole et d'une alimentation de proximité, etc. De nouvelles agglomérations sollicitent aussi « Du pré au plat» pour un approvisionnement local de leurs restaurants scolaires.

Par ailleurs, plusieurs autres démarches, alors en cours de lancement sur le territoire (une CUMA d'abattage de volailles, l'opération « terroirs sur la route »), vont devenir parties prenantes de « Du pré au plat» (jusqu’à être intégrée au collectif pour « terroirs sur la route »).

Les nouveaux projets ne manquent pas non plus. La CUMA développe l'activité logistique de tournée de livraison, de nouveaux coopérateurs arrivent suite à la fermeture d'un site de transformation. Parallèlement, les éleveurs associés lancent une réflexion sur les relations possibles 
avec les artisans-commerçants du territoire. Pour eux, le développement de l'atelier et plus largement de la vente directe par les producteurs, ne doit pas se faire au détriment du développement économique local en particulier pour les boucheries-charcuteries du territoire. La rencontre des deux corps de métier est complexe. Il faut échanger sur les contraintes mutuelles, les attentes, etc., pour apprendre à se connaître et reconnaître les savoir-faire de chacun. Evènement marquant de ce renouveau, le projet d'abattoir pensé lors de la création est relancé. «Du pré au plat » constitue désormais une offre de services à destination de tous les producteurs du territoire et le projet est moteur d'une gouvernance alimentaire territoriale en construction (synergies avec des artisans, approvisionnement de la restauration locale, projet d'abattoir). Aussi, une modification du statut juridique vers une SCIC est envisagée afin d'intégrer les collectivités.

Figure 3 - L'articulation des projets durant la phase de renouvellement

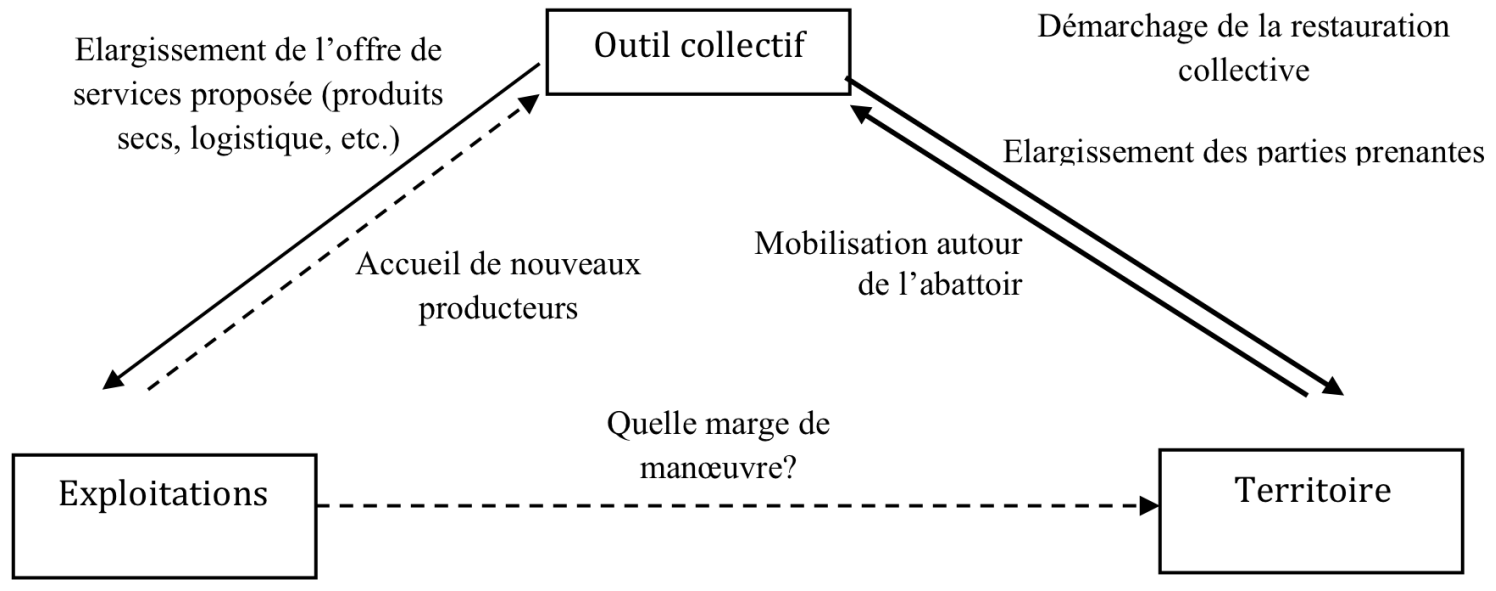

\section{Discussion et conclusion}

Les collectifs en circuits courts constituent des formes d'organisation collective spécifiques qui mobilisent sur un territoire géographique restreint une pluralité d'acteurs - producteurs, accompagnateurs, collectivités locales et quelques fois les consommateurs. L'ensemble de ces acteurs sont engagés dans la construction de nouvelles coordinations territoriales fondées sur la coopération et la mutualisation. Comme nous l'avons vu, ces initiatives constituent d'authentiques stratégies collectives coopératives (Loup, 2007), dans lequel le projet collectif a à la fois une 
dimension fortement opérationnelle et stratégique, puisqu'il s'agit d'élargir les débouchés des producteurs, et une dimension politique de transformation du champ agricole. Par contre, la pérennisation de ces systèmes n'est pas facile à obtenir, tant ils mobilisent des niveaux d'action complexes - le niveau micro de l'exploitation, le niveau meso du projet et le niveau plus macro du territoire institutionnel et des acteurs aux ressources économiques limitées. Alors que les initiatives continuent de se développer, à travers des formes originales et innovantes et contribuent à créer des emplois et favoriser l'ancrage territorial des activités agricoles, les questions de gestion des relations entre ces différents niveaux d'action mais également entre les individus investis dans ces projets complexes se pose avec insistance, jusqu'à éclatement de la dynamique parfois. Le cas du Pré du plat constitue à cet égard une opportunité de recherche importante riche d'enseignements car il constitue une de ces initiatives collectives relativement ancienne dont on peut retracer la trajectoire.

L'analyse du cas Du pré au plat révèle en premier lieu le caractère profondément instable de ce type de stratégies collectives ; elle confirme notamment les difficultés organisationnelles et stratégiques que les acteurs rencontrent (Leyronas et Loup, 2008). Les facteurs de déséquilibre sont effectivement nombreux et différents aux étapes successives d'émergence et de développement du projet commun. Ainsi, ils sont liés à des facteurs macro-économiques (comme la hausse du carburant) mais également à des difficultés à constituer des ressources collectives propres qui ont généré ou accentué les difficultés organisationnelles: dissensus sur l'intensité de l'engagement dans le collectif, importants coûts de coordination. Ce sont également des difficultés à accorder et faire évoluer de façon cohérente, stratégies individuelles et collectives. Aussi, à mesure que l'activité a été développée, le projet collectif initial a lui-même fortement évolué, tant au niveau de la stratégie de commercialisation (élargissement des débouchés commerciaux vers la GMS et la restauration collective, et des gammes) que de la transformation (face aux difficultés financières, l'enjeu de productivité du travail est devenu prégnant). De même, le caractère politique de la stratégie collective du Pré au plat, qui s'inscrit dans le champ des alternatives agricoles (Lanciano et 
Saleilles, 2014) a certainement contribué à faire émerger le projet, mais il explique aussi la polarisation de certaines positions et la forme prise par certains échanges. Ainsi, certains producteurs, notamment les fondateurs, ont pu craindre un basculement vers le «tout économique » alors que le projet initial avait une forte dimension militante : développement social et local. Dans le champ de ces alternatives agricoles et alimentaires, l'enjeu n'est plus véritablement aujourd'hui de favoriser l'émergence de nouvelles initiatives mais plutôt de résoudre les problèmes de coordination que pose leur développement.

Mais l'analyse particulière de « Du pré au plat » s'avère également riche d'enseignements, dans la façon dont la démarche a, malgré tout, perduré. L'analyse de sa trajectoire historique maintenant assez significative, révèle en réalité des facteurs de stabilité, qui font directement intervenir le territoire. Nous avons développé une approche en terme d'équilibre instable entre trois variables : projet d'exploitation - projet collectif - projet de territoire. Ces trois dimensions constituent tout à la fois les raisons d'être du projet collectif que des ressources pour rétablir, parvenir ou relancer la dynamique collective, aux différents moments de sa trajectoire. Ainsi, si lors de la naissance et de l'ascension, l'accent est mis sur l'équilibre entre projets d'exploitation et collectif, la phase de remise en cause va progressivement conduire le collectif à valoriser le territoire dans ses différentes dimensions. Au fil des étapes et des épreuves traversées, on observe en fait des modifications dans l'équilibre entre les trois dimensions inhérentes au projet : le projet d'exploitation, le projet collectif centré sur l'outil et le projet de territoire dans lequel les acteurs institutionnels locaux sont associés. L'articulation entre ces trois dimensions, différente suivant les circonstances, constitue un facteur de stabilisation et de pérennisation de la dynamique collective. Le triptyque est alors réinterrogé et réinventé sans cesse pour mobiliser les élus, les producteurs et les consommateurs, avoir une vision stratégique (et pas juste fonctionnelle du projet) et des perspectives d'innovation et de pérennisation. Au cours de ces ré-articulations, le territoire, dans ses différentes dimensions, est alors utilisé comme un outil de stabilisation de la dynamique collective. Il convient alors de voir comment ces modifications d'équilibre ont été opérées et dans quelles mesures ils constituent des 
ressources pour les acteurs pour rétablir la dynamique collective. De ce point de vue, notre analyse révèle l'importance des niveaux différents d'action et des parties prenantes comme des ressources pertinentes pour le développement des stratégies collectives. Elle invite ainsi à intégrer le territoire comme une véritable ressource dans l'analyse des stratégies collectives. Le territoire ne représente en effet pas seulement l'espace de l'action publique, ni même une ressource patrimoniale. Il constitue une ressource que les acteurs peuvent mobiliser, pour résoudre notamment la tension entre les niveaux individuel et collectif.

L'analyse des facteurs de réussite et de faiblesse du cas du Pré au Plat en termes de trajectoire amène finalement à penser les modalités d'accompagnement de ces systèmes. Comme nous l'avons vu, il ne s'agit pas seulement de se concentrer sur des bonnes pratiques mais aussi de repérer les ressources et les stratégies d'adaptation qui peuvent être réalisées dans les situations de déséquilibre. Enfin, ce cas souligne le rôle clé de l'accompagnateur et de producteurs fondateurs qui dès le départ avaient une volonté de faire en sorte que l'outil commun soit un moteur du développement agricole et local. En ce sens, des travaux complémentaires sont sans doute nécessaires pour mieux cerner le rôle de ces entrepreneurs dans le développement du triptyque.

\section{Références bibliographiques}

ASSELINEAU, Alexandre et CROMARIAS Anne, Les stratégies collectives sont-elles toujours applicables dans un « milieu »? Une réflexion à partir du contre exemple de la coutellerie thiernoise, Management \& Avenir, 2011, vol. 10, n 50, p. 137-152.

ASSELINEAU, Alexandre et CROMARIAS Anne. Entreprise et territoire, architectes conjoints d'un développement local durable ?, Management \& Avenir, 2010, vol. 6, n 36, p. 152-167.

ASTLEY, Graham W. et FOMBRUN, Charles J. Collective strategy: social ecology of organizational environments, Academy of Management Review, 1983, vol. 8, n 4, p. 576-587. BRESSER, Rudy. Matching Collective and Competitive Strategies. Strategic Management Journal, 1988, vol. 9 , n 4 , p. $375-385$ 
DUFOUR, Annie et LANCIANO, Émilie. Les circuits courts de commercialisation : un retour de l'acteur paysan ?, Revue Française de Socio-Économie, 2012, vol.1, n 9, p. 153-169.

FOURCADE, Colette. Les systèmes agroalimentaires comme modalités collectives. Revue Française de Gestion, 2006, vol.8, n 167 , p.183-201.

FOURCADE, Colette. Des dynamiques territorialisées novatrices: le cas des PME agroalimentaires, Revue d'Économie Régionale \& Urbaine, 2008, vol. 2, p. 193-210

GRANATA, Julien. et LE ROY, Frédéric. Le management de la coopétition en PME : le cas des vignerons du Pic Saint-Loup, Finance Contrôle Stratégie, 2014, vol. 17 n²

GUNDOLF, Katherine et JAOUEN, Annabelle (dir.). Les relations interorganisationnelles des PME, 2008, Paris, Hermès-Lavoisier,

GUNDOLF, Katherine et JAOUEN, Annabelle, Emergence et pérennité des stratégies collectives territorialisées: le rôle de l'entrepreneuriat collectif, MESSEGHEM, Karim., POLGE, Marion. et TEMRI, Leïla. (éds), Entrepreneur et dynamiques territoriales, 2009, Paris, EMS, 291-306.

GUNDOLF, Katherine, JAOUEN, Annabelle et LOUP, Stéphanie. Institutions locales et TPE dans le cas du tourisme, Revue Française de Gestion, 2006, vol. 8, n¹67, p. 141-155

GUMUCHIAN, Hervé et PECQUEUR Bernard. La ressource territoriale, 2007, Ed. Economica, Anthropos, coll. Géographie, Paris, 252 p.

LANCIANO, Emilie et SALEILLES, Séverine. Le développement des circuits courts alimentaires : un nouveau souffle entrepreneurial dans l'agriculture?, Congrès International Francophone sur l'Entrepreneuriat et la PME, 2010 Bordeaux, France.

LANCIANO, Emilie et SALEILlES, Séverine., Processus de construction d'un champ d'innovation: le cas de l'alimentaire alternatif dans la métropole lyonnaise, Journées Doriot «Entrepreneuriat et Société », 2014, Rabat, 15-16 mai

LE CARO, Yvon et DANIEL, Ronan. Les motivations des agriculteurs en vente directe en Bretagne, In AMEMIYA, Hiroko (ed.), L'agriculture participative. Dynamiques bretonnes de la vente directe. 2007, Rennes, PUR. 
LEYRONAS, Christophe et LOUP, Stéphanie. Cadre d'analyse des effets de leviers des stratégies collectives entrepreneuriales en TPE, $9^{\circ}$ colloque International Francophone en Entrepreneuriat et PME, 2008, Louvain-la-Neuve, Belgique.

LOUBARESSE, Elodie et PESTRE, Florent. Les facteurs de réussite d'une stratégie collective hybride : le rôle de l'acteur tiers, 21ème Conférence de l'Association Internationale de Management Stratégique, 2012, Lille, France.

LOUP, Stéphanie. La stratégie coopérative : un cas particulier de stratégie collective, In LE ROY, Frédéric et YAMI, Saïd (coord.), Les stratégies collectives - Rivaliser et coopérer avec ses concurrents, 2007, EMS Management \& Société

MONDY, Bernard et TERRIEUX Agnès. Vers une agriculture territorialisée. Où s'alimentent les circuits courts ? Le rôle des ateliers collectifs de transformation agroalimentaire, in Circuits courts : contribution au développement régional, TRAVERSAC, Jean-Baptiste (éds.), 2011, Educagri, Dijon, p. 89-101.

MUNDLER, Patrick et VALORGE, Fabien. Ateliers de transformation collectifs : enjeux et outils pour réussir, 2015, Quaé.

SANZ CANADA, Javier et MUCHNIK, José. Ancrage et identité territoriale des systèmes agroalimentaires localisés, Economie Rurale, 2011, n³22, p. 4-10.

THOMAS, Franck. SCIC et agriculture. Le temps des défricheurs, RECMA - Revue Internationale de l’Economie Sociale, 2009, n³10, p.17-30

WACHEUX, Frédéric. Méthodes qualitatives et recherche en gestion. Paris, Economica, 1996 YIN, Robert. K. Case study research. Design and methods. Londres, Sage Publications, 1994 